\title{
Psicosinergia en Millon: Del modelo Biopsicosocial al modelo Ecológico
}

\section{Psychosynergy in Millon: From Bio-psycho-social model to Ecological model}

\author{
Jesús R. Herrero Sánchez* \\ Universidad de Salamanca, España
}

(Recepción: Octubre 2007 - Aceptación: Noviembre 2007)

\begin{abstract}
Resumen
Desde la publicación de Psicopatología Moderna, el modelo psicopatológico de Millon ha evolucionado sustantivamente. En su forma actual, Millon afirma que la estructura de una ciencia clínica consiste en cuatro elementos principales: una teoría que explica los fenómenos bajo observación, (b) una taxonomía que categoriza estos fenómenos en dimensiones significantes, (c) una instrumentación que mide estos fenómenos, y (d) una intervención que permite tratar los casos problemáticos. Este artículo analiza el primer elemento: la teoría. Dicha teoría se traduce en un desarrollo personológico basado en la teoría para tanto los estilos de personalidad normal como para la personalidad patológica.

Palabras claves: Personalidad, trastornos de personalidad, Millon, psicopatología, modelo biopsicosocial, modelo evolucionista, modelo ecológico.
\end{abstract}

\begin{abstract}
Since the publication of Modern Psychopathology, Millon's model of psychopathology evolved and expanded. In its current form, Millon asserts that the structure of a clinical science consists of four main elements: (a) a theory that explains the phenomena under observation, (b) a taxonomy that categorizes these phenomena into meaningful dimensions, (c) instrumentation that measures these phenomena, and (d) intervention that remediates problematic cases. This paper analizes the first element: Theory. This theory of personology development translates into a theory-based framework for both personality styles and personality pathology.

Key words: Personality, personality disorders, Millon, psychopathology, bio-psycho-social model, evolutionary model, ecologic model.
\end{abstract}

\footnotetext{
* Correspondencia a: Jesús R. Herrero Sánchez. Dpto. Personalidad, Evaluación y Tratamiento Psicológicos, Facultad de Psicología, Universidad de Salamanca. Avda. La Merced 109-131, 37005 - Salamanca, España. Teléfono: 923294500 Ext. 3319 E-Mail: jherrero@usal.es
} 


\section{Introducción y tesis}

Los trastornos de personalidad, a pesar de reconocerse su existencia e importancia desde tiempos remotos, no se han convertido en un tema principal de interés de la investigación, la teoría y la práctica clínica, hasta tiempos muy recientes. Su aparición en el DSM-III (APA, 1980) provocó una gran corriente de interés y la publicación de numerosas investigaciones, convirtiéndose en objeto de preocupación central de muchos clínicos e investigadores. Aunque la naturaleza exacta de los trastornos de personalidad no sea demasiado evidente, a menudo está claro que existe un problema, indicado por la conducta del paciente en cuestión.

A través de una variadísima colección de escritos Millon pone de manifiesto todos sus esfuerzos por construir una ciencia unificada entre Personología y Psicopatología. Desde la primera formulación del modelo (Millon, 1969/1976) hasta nuestros días se han sucedido un conjunto ordenado y articulado de proposiciones teóricas (Millon, 1981, 1984/1992, 1986a, 1986b, 1990; Millon y Millon, 1974; Millon y Everly, 1985/1994), también descritas en nuestro contexto (Ávila y Herrero, 1995, 1997; Ávila, Rodríguez y Herrero, 1997; Kirchner, Torres y Forns, 1998), las cuales han permitido no sólo describir sino también teorizar la personalidad, mediante la combinación de modelos biológicos, dinámicos, funcionales, cognitivos e interpersonales en una misma sistemática teórica, con la ventaja añadida de no renunciar a todo lo que encuentra de valioso en tradiciones teóricas anteriores.

La propuesta de Millon se estructuraría en los siguientes términos (Millon y Davis 1996, 1997; Millon, 1991, 1999a): una ciencia clínica verdaderamente madura consiste en cuatro dominios (teoría, taxonomía, evaluación e intervención) interconectados entre sí. Además, todas las actividades de los clínicos, desde un punto de vista científico, estarían guiadas hacia uno o más de estos dominios clínicos. El dominio de la teoría estaría formado por esquemas conceptuales, explicativos y heurísticos, que habilitarían su desarrollo formal. Consistiría en un cuerpo de proposiciones que darían cuenta de distintos constructos de la personalidad y la psicopatología para estudiar las realidades clínicas. Es decir, poner en relación una serie de principios científicos universales, guiados por las leyes naturales de la evolución, con un marco teórico orientado a la persona o personología.

La taxonomía pondría en juego categorías y/o dimensiones dentro de las cuales la personalidad y los fenómenos psicopatológicos podrían ser valorados y clasificados. Consistiría en una clasificación de patologías (o nosología) donde, a partir de una tipología y sus rasgos característicos derivados de la teoría, se pondrían en relación categorías diferenciadas, agrupadas e interrelacionadas.

La evaluación o instrumentación clínica tendría que ver con la descripción y explicación de la patología individual; tales instrumentos, cualitativamente sensibles y diseñados desde una base empírica, tratarían de identificar las categorías diagnósticas y medir los rasgos característicos de las personas.

Finalmente, la intervención (o tratamiento) buscaría acomodar y remediar las patologías, mediante la especificación de objetivos, la planificación de estrategias y la selección de modalidades de tratamiento apropiadas para cada aspecto de la persona. Un esquema de este tipo es denominado por Millon como "Psicosinergia" $(1990,1999$ a) y pretende dar coherencia a los elementos de una ciencia clínica. Este artículo analiza el primer elemento: la Teoría.

\section{Discusión}

La personalidad en Millon (Millon y Everly, 1985/1994) representa un patrón profundamente arraigado en el individuo, compuesto por cogniciones, afectos y conductas, que persisten por largos periodos de tiempo, donde el aprendizaje experiencial y las disposiciones biológicas juegan un importante papel (Millon, 1969/1976).

La personalidad emerge como resultante de la interdeterminación de dos procesos: cómo el individuo interactúa con las demandas de su medio ambiente y cómo se relaciona consigo mismo. $\mathrm{Si}$ el complejo proceso evolutivo de determinaciones biológicas e interpersonales que atraviesa el individuo da lugar a que se manifieste un patrón de personalidad caracterizado por que el individuo 
manifieste habilidad para abordar su entorno con flexibilidad y capacidad de adaptación, tolerante ante la frustración y aceptando demora en la gratificación, de manera que sus comportamientos y percepciones característicos fomenten el aumento de gratificaciones personales, entonces podemos hablar de normalidad (Ávila y Herrero, 1995). Esta "normalidad" se concreta en una actitud constructiva ante el entorno y en comportamientos promotores de salud.

Si por el contrario el individuo muestra escasa flexibilidad para adaptarse, lo que se traduce en una falta o escasez de estrategias alternativas en la relación con los demás, utilizándolas de forma rígida, y es incapaz de adaptarse a los cambios, tendiendo a modificar su entorno para que éste no le exija comportamientos que están fuera de su repertorio, entonces hablaremos de patrón de personalidad patológico (Millon, 1986a, 1986b). En él encontraremos además la tendencia a crear círculos viciosos que se manifestarán como patrones repetitivos, y en algunos casos, los más disfuncionales, el patrón de personalidad será inestable, a causa de la debilidad del Yo, y dará lugar a un patrón de personalidad patológico "por naturaleza" o que es fácilmente observable como tal en cualquiera de sus ámbitos de manifestación. Esta rigidez y repetitividad del patrón indicará la exacerbación patológica de un estilo de personalidad que podría haber sido normal de ser más flexible, mientras que la inestabilidad del patrón dominante indica una disfunción más grave (p.e. las personalidades esquizotípica, límite o paranoide).

Para Millon los Trastornos de Personalidad son extensiones patológicas de los patrones de personalidad normal; dicho de otro modo, “... emergen de los patrones de personalidad normal como resultado de interacciones complejas de disposiciones biológicas, aprendizaje desadaptativo y especialmente cambios ambientales estresantes" (Millon y Everly, 1985/1994).

Ahora bien, el desarrollo de la teoría de Millon ha tenido dos etapas importantes para la definición de los patrones de personalidad. En una primera etapa se basó de forma predominante en la idea de las estrategias de afrontamiento y las fuentes de refuerzo (Millon, 1969/1976, 1981), y en la segunda etapa ha integrado esta idea con los principios de la teoría de la evolución y de la ecología (Millon, 1990). La unión de estas dos teorías, es uno de los aspectos más originales y distintivos en la obra de Millon.

\section{El modelo biopsicosocial}

Como ya se ha mencionado unas líneas más atrás, el aprendizaje experiencial y las disposiciones biológicas juegan un importante papel en la constitución de la personalidad. Además, muestran una estrecha relación con la construcción de la teoría del aprendizaje biopsicosocial (Millon, 1969/1976).

Esbozada ya una primera versión del modelo en Psicopatología Moderna (1969/1976), es en 1981 cuando aparece desarrollada con profundidad una teoría sobre el desarrollo de la personalidad, en la que se acepta una interacción compleja entre bases biológicas, procesos evolutivos y peculiaridad de los aprendizajes. De forma complementaria, y como resultante de la determinación que ejercen las diversas fuentes de influencia, Millon sostiene que se habría ido definiendo un "estilo de personalidad" y construyendo un "patrón de personalidad" sobre la base de la calidad de las experiencias interpersonales que el sujeto tiene a lo largo de su vida, aunque principalmente en las primeras etapas (Ávila y Herrero, 1995).

Tomando como premisa de partida los factores biológicos y ambientales, Millon (1969/1976, 1981, 1986a) explica teóricamente el origen de los patrones o pautas de personalidad sobre la base de dos dimensiones:

1) Una dimensión de "acción" (Actividad versus Pasividad), entendida como patrón básico de reforzamiento instrumental que el sujeto emplea para maximizar la obtención de los refuerzos y minimizar el dolor o sufrimiento asociado a su logro: Actividad: búsqueda activa de refuerzo (proactivo); y Pasividad: búsqueda pasiva de refuerzo (reactivo). Se trata de una dimensión anclada en lo biológico (individualidad biofísica del sujeto) y modelada por el aprendizaje instrumental. 
2) La dimensión "clase de vínculo interpersonal entre el Sí mismo y los Otros”, estructurada en 4 categorías (Retraimiento, Independencia, Dependencia y Ambivalencia), dimensión entendida como objetivación resultante del desarrollo psicológico de la fuente u origen primario de las satisfacciones y refuerzos que el sujeto logra. Cada una de las categorías implica niveles cualitativamente diferentes de integración de las experiencias interpersonales: Retraimiento: Escasa relación del sujeto consigo mismo o con los otros, con tendencia al aislamiento y desinterés hacia lo interpersonal. Es descrita como estilo Desvinculado; Dependencia: Los sujetos evalúan sus experiencias de satisfacción o malestar en función de cómo los otros reaccionan o sienten hacia ellos; es decir, el sujeto depende del objeto; Independencia: Los sujetos obtienen gratificación por sus propios valores o deseos, con escasa referencia a los otros; es decir, el sujeto es autosuficiente y ensaya la independencia del objeto, que puede connotarse negativamente; $\mathrm{Am}$ bivalencia: Los sujetos oscilan entre la pauta de dependencia e independencia, bien utilizando ambas pautas, bien recurriendo cíclicamente a una u otra. Es descrito como estilo Confuso.

El cruce de estas dos dimensiones teóricas puede ser representado como dos ejes ortogonales en los que la evolución del individuo hacia un patrón de mayor complejidad estaría representado hipotéticamente por un vector que cruza el origen en un ángulo de $45^{\circ}$, sugiriendo que la evolución normal es un producto de la influencia de la individualidad biofísica y de las experiencias de aprendizaje interpersonal.

\section{La perspectiva evolucionista}

En 1990, Millon reconceptualiza su teoría, identificando tres polaridades evolutivas como un modelo más amplio para explicar el origen y construcción de la personalidad. Su pensamiento deductivo muestra que los principios y procesos de la evolución son esencialmente universales, expresados, por supuesto, en una variedad de esferas diferentes, que abarcan tanto a la física como a la química, la biología o la psicología, impregnando a su paso todo el funcionamiento humano (Davis, 1999).

Ahora las polaridades operan como "atractores" y organizadores de las experiencias de aprendizaje interpersonal, y concretan la posibilidad evolutiva de que un patrón de personalidad quede fijado. Tres son las polaridades propuestas: Dolor-Placer (la más primitiva, anclada en lo biológico), Actividad-Pasividad (transición de lo biológico a psicológico), Sí mismo-Otros (plano psicológico y psicosocial).

Así la personalidad, normal o patológica, es concebida como una interacción compleja de funciones y estructuras psíquicas, en torno a las tres polaridades cuya finalidad existencial puede ser descrita en los siguientes términos (Millon, 1990; Ávila y Herrero, 1995):

1) Preservar y potenciar la vida, mediante la maximización del "Placer" y la minimización del "Dolor". Tanto el "Placer" como el "Dolor" son dimensiones separadas bipolares, de manera que una persona puede ofrecer valores altos o respuestas características del polo alto o bajo, bien en una de ellas o en ambas, ya que son conceptos antitéticos, por ejemplo el "Placer" no puede ser definido como un nivel bajo o mínimo de "Dolor", ni a la inversa.

2) Emplear modos de conducta y pensamiento que maximicen la adaptación al entorno, mediante el logro de un balance óptimo entre la "acomodación pasiva" y la "modificación activa". "Pasividad" y "Actividad" forman una dimensión unipolar, ya que implican en lo esencial diferencias cuantitativas en un continuo o gradiente de rendimiento o actividad motora.

3) Lograr estrategias conductuales, de emoción y pensamiento que maximicen el éxito repetido en lograr un balance óptimo entre desarrollo e integración del "Sí mismo" y el acogimiento por los "Otros". "Sí mismo" y los "Otros" son también dos dimensiones bipolares independientes y antitéticas conceptualmente, en las que, por ejemplo, que el foco de interés esté puesto en los "Otros" no excluye el interés en el "Sí mismo".

Las posibilidades de covariación e interacción entre todas estas dimensiones son mucho más amplias que las que recoge descriptivamente el modelo de polaridades evolutivas a la hora de su 
concreción en prototipos de personalidad normal y de trastornos de la personalidad, por lo que no cabe descartar revisiones parciales o nuevas propuestas (véase p.e. Millon, 1994).

Sobre estos principios se desarrolla este modelo evolutivo, en el que las dimensiones previas son revisadas, incluyéndose una categoría más en la dimensión "origen" o fuente primaria de la que el sujeto obtiene la gratificación o evita el dolor o malestar emocional. A las ya descritas categorías de Retraimiento, Dependencia, Independencia y Ambivalencia, se añade ahora la de Discordancia, entendida como incapacidad para objetivar la fuente primaria, que se traduce en una variante disfuncional o fallida de las otras categorías. Discordancia implica déficit estructural en la posibilidad de objetivación de la relación Sí mismo-Otros.

\section{La perspectiva ecológica}

Pocas líneas más atrás se apuntaba la posibilidad, quien sabe si la necesidad, de que en el modelo hubiese lugar para revisiones parciales o nuevas propuestas. De hecho Millon ha mantenido desde 1981 un especial compromiso de acercar sus posiciones teóricas a la evolución del consenso científico y profesional que preside los criterios diagnósticos de la Asociación Psiquiátrica Americana en sus últimas cuatro versiones, desde el DSM-III (APA, 1980) hasta el DSM-IV-TR (APA, 2000). Es cierto que esta postura puede haber implicado en algún momento un sometimiento excesivo a exigencias que no se derivan directamente de la teoría (Ávila y Herrero, 1995), pero si han contribuido a enriquecer aún más su modelo teórico.

Los argumentos que conectan las teorías ecológica y evolutiva están bien establecidos y ofrecen alguna justificación por extender los principios de la adaptación a los estilos humanos (Davis, 1999). Para proporcionar un fondo conceptual al vasto mundo de los estilos y patrones de personalidad, Millon utiliza de forma metafórica los cuatro dominios en los que se demuestran los principios evolutivos y ecológicos, como son, la finalidad de la existencia, el modo de adaptación, las estrategias de replicación y el grado de abstracción. En este sentido, la meta de coordinación teórica podría lograrse, según Millon, explorando el poder de un modelo evolutivo para simplificar y poner en orden rasgos personológicos previamente dispares.

Mediante el principio finalidad de la existencia, los seres vivos tienden sucesivamente a su transformación desde estados caóticos o de escasa organización hasta otros de mayor complejidad, en un intento de perfeccionamiento y preservación de la vida. En términos de la teoría de Millon equivale a definir la polaridad Placer-Dolor; es decir, reconocer y seguir sensaciones y emociones positivas, o reconocer y evitar sensaciones y emociones negativas. El equilibrio o desequilibrio de un polo sobre el otro, así como el posible conflicto entre ambos expresarán diferentes formas psicopatológicas.

Por el principio evolutivo de la adaptación se expresan los procesos homeostáticos o de equilibrio de los organismos. En términos de Millon este principio constituye la segunda polaridad, Actividad-Pasividad. Este principio evolutivo pone en relación lo que Millon denomina modos de adaptación: modificación y acomodación ecológicos.

El primer modo, la acomodación ecológica, significa una inclinación pasiva, servicial y dependiente en relación con el contexto; supone un medio de supervivencia en el que los elementos que constituyen el ambiente del sujeto proporcionan a éste protección y sustento para su existencia. Contrasta con éste el segundo de los modos de adaptación, la modificación ecológica, que implica una tendencia a cambiar o reestructurar los elementos que constituyen el entorno.

El principio evolutivo de la replicación está en relación con la polaridad Sí mismo - Otros y expresa la dirección en la que el sujeto busca sus refuerzos positivos. La personalidad, bajo la perspectiva de esta polaridad, puede desarrollarse dando preferencia y prioridad a "los demás", o al "sí-mismo", o adoptando una posición de conflicto entre ambas polaridades.

Finalmente, el cuarto principio es la abstracción, la capacidad de simbolizar el mundo de uno mismo. Es decir, tiene que ver con la emergencia de competencias que promueven la planificación anticipatoria y la toma razonada de decisiones en el sujeto (Kirchner, Torres y Forns, 1998). 


\section{Conclusiones}

Conceptualmente, resulta conveniente examinar la personalidad humana como el fundamento básico en el que la conducta transituacional puede ser reconstruida desde una perspectiva más iluminadora e integrativa. En último término, esta perspectiva revela una serie de patrones y rasgos que introducen un orden y un sentido a las que previamente parecían tan sólo ser conductas contingentes con la situación y sin interrelación. Por tanto, una verdadera formulación multidimensional de la personalidad nos puede proporcionar un instrumento poderoso para comprender, predecir e incluso alterar la conducta humana en toda su diversidad.

Evidentemente, el modelo propuesto en este artículo no es único. Dos mil años de historia de la humanidad tienen en nuestro campo de estudio un factor común e inequívoco: la humanidad ha estado empeñada en un esfuerzo de comprensión. Y quizás por esta razón, el constructo de la personalidad ha sido formulado, reformulado y discutido durante siglos.

De entre los diversos modelos formulados para la formación, desarrollo y manifestación de la personalidad -y sus trastornos- se encuentra la teoría evolucionista desarrollada desde finales de los sesenta por Theodore Millon, con un enfoque capaz de aunar modelos biológicos, dinámicos, funcionales, cognitivos e interpersonales en una sistemática teórica que permite no sólo describir sino también teorizar la personalidad. Las derivaciones valorativas que han surgido dentro de esta perspectiva son numerosas y nos permiten a su vez aplicar los conceptos a planteamientos de evaluación y su concreción en numerosos instrumentos de exploración de la personalidad.

\section{Referencias}

American Psychiatric Association (1980). Diagnostic and statistical manual of mental disorders. (DSM-III ). Washington, DC: APA.

American Psychiatric Association (2000). Diagnostic and statistical manual of mental disorders (DSM-IV-TR). Washington, DC: APA.

Ávila, A. y Herrero, J. R. (1995). La Personalidad y sus Trastornos: Aproximación a la obra de Theodore Millon. Clínica y Salud, 6, 131-159.

Ávila, A. y Herrero, J. R. (1997). Tendencias actuales en evaluación de la personalidad y sus correlatos clínicos. Revista Portuguesa de Psicologia, Año 1995/1996, 141-171.

Ávila, A., Rodríguez, C., y Herrero, J. R. (1997). Evaluación de la personalidad patológica. Cap. 4 de la obra de A. Cordero (Coord.), La evaluación psicológica en el año 2000 (pp. 79-107). Madrid: TEA Ediciones, S.A.

Davis, R. D. (1999). Millon: Essentials of his science, theory, classification, assessment, and therapy. Journal of Personality Assessment, 72, 330-352.

Kirchner, T., Torres, M. y Forns, M. (1998). El modelo de polaridad de Millon, en T. Kirchner, M. Torres, y M. Forns (Eds.), Evaluación psicológica: modelos y técnicas (pp. 241-266). Barcelona: Paidós.

Millon, T. (1976). Psicopatología Moderna: Enfoque biosocial de los aprendizajes erróneos y de los disfuncionalismos [Modern psychopathology]. Barcelona: Salvat. (Original publicado en 1969)

Millon, T. (1981). Disorders of Personality: DSM-III, Axis II. Nueva York: Wiley.

Millon, T. (1992). Sobre el renacimiento de la teoría y la evaluación de la personalidad [On the renaissance of personality assessment and personality theory]. En A. Avila-Espada, y C. Rodríguez-Sutil, (Eds.), Psicodiagnóstico Clínico, 2a edición revisada (pp. 118-124). Madrid: Eudema. (Reimpreso de Journal of Personality Assessment, 1984, 48, 450-466).

Millon, T. (1986a). A theoretical derivation of pathological personalities. En T. Millon y G. L. 
Klerman (Eds.), Contemporary directions in psychopathology: Toward the DSM-IV (pp. 639670). Nueva York: Guilford Press.

Millon, T. (1986b). Personality prototypes and their diagnostic criteria. En T. Millon y G. L. Klerman (Eds.), Contemporary directions in psychopathology: Toward the DSM-IV (pp. 671-712). Nueva York: Guilford Press.

Millon, T. (1990). Toward a new personology: An evolutionary model. Nueva York: John Wiley and Sons.

Millon, T. (1991). Normality: What may we learn from evolutionary theory? En D. Offer and M. Sabshin (Eds.), The diversity of normal behavior: Further contributions to normatology (pp. 356-404). Nueva York: Basicbooks, Inc.

Millon, T. (1994). Personality Disorders: Conceptual distinctions and classification issues, en Paul T. Costa Jr. y Thomas A. Widiger (Eds.) Personality disorders and the five-factor model of personality (pp. 279-301), Washington, DC: APA.

Millon, T. (1999a). Reflections on psychosynergy: a model for integrating science, theory, classification, assessment and therapy. Journal of Personality Assessment, 72, 437-456.

Millon, T. y Davis, R. D. (1996). An evolutionary theory of personality disorders. En J.F. Clarkin y M.F. Lenzenweger, (Eds.), Major theories of personality disorders. Nueva York: The Guilford Press.

Millon, T. y Millon, R. (1974) Psicopatología y Personalidad [Abnormal Behavior and Personality] Mexico: Interamericana. (Trabajo original publicado en 1974).

Millon, T. y Everly, G. S. (1994). La personalidad y sus trastornos [Personality and its disorders: A biosocial learning approach]. Barcelona: Martínez Roca. (Trabajo original publicado en 1985). 